\title{
RESPUESTAS DE LOS PAÍSES DEL PACÍFICO SURAMERICANO ANTE LA MIGRACIÓN VENEZOLANA: ESTUDIO COMPARADO DE POLÍTICAS MIGRATORIAS EN COLOMBIA, ECUADOR Y PERÚ
}

\author{
RESPONSES OF THE COUNTRIES OF THE SOUTH AMERICAN PACIFIC \\ REGARDING VENEZUELAN MIGRATION: COMPARATIVE STUDY OF \\ MIGRATION POLICIES IN COLOMBIA, ECUADOR AND PERU
}

\author{
Nicolás Gissi Barbieri*, Jacques Ramírez Gallegos**, María del Pilar Ospina Grajales***, \\ Bárbara Pincowsca Cardoso Campos ${ }^{* * * *}$ y Sebastián Polo Alvis*****
}

\begin{abstract}
El objetivo del artículo es analizar el flujo migratorio procedente de Venezuela hacia Colombia, Ecuador y Perú en el periodo 2015-2019. Se realiza un análisis comparado de la evolución de las políticas migratorias de los tres países, a partir de una revisión legislativa, análisis de políticas y medidas institucionales adoptadas. Si bien hay una emergente coordinación política a nivel regional, también hay particularidades en las respuestas construidas en los países estudiados. La migración venezolana se ha transformado en un hecho prioritario en la subregión del Pacífico Sur, implementándose políticas específicas, pero no suficientes. El análisis de los tres países examinados da cuenta de la implementación de un enfoque de gestión de las migraciones que ha mutado (cada vez que hay estampidas migratorias) hacia enfoques restrictivos de seguridad y control.
\end{abstract}

Palabras claves: migración venezolana, Pacífico Suramericano, crisis humanitaria, políticas públicas.

The article's objective is to analyze the migratory flow from Venezuela to Colombia, Ecuador, and Peru in the period $2015-2019$. A comparative analysis is made of the evolution of migration policies in the three countries, based on a legislative review, policy analysis, and institutional measures adopted. While there is an emerging policy coordination at the regional level, there are also particularities in the responses built in the countries studied. Venezuelan migration has become a priority in the South Pacific sub-region, with specific but not sufficient policies being implemented. The three countries' analysis shows the implementation of a migration management approach that has mutated (each time there are migratory stampedes) towards restrictive approaches of security and control.

Key words: Venezuelan migration, South American Pacific, humanitarian crisis, public policies.

\section{Introducción}

La salida masiva de personas desde Venezuela es considerada como una de las crisis migratorias más relevantes en América Latina en los últimos tiempos (Oficina del Alto Comisionado de las Naciones Unidas para los Derechos Humanos [OACNUDH] 2018, Human Rights Watch 2018). Aproximadamente cuatro millones de personas han abandonado Venezuela hasta finales del 2019 según la Organización de los Estados Americanos (OEA). La rapidez y la magnitud de esta movilidad internacional han desbordado la capacidad de respuesta institucional de los Estados de la región y abrieron un llamado a la revisión y puesta en práctica de políticas transitorias en materia de migración (Banco Mundial 2018).

El objetivo del presente artículo consiste en realizar un análisis comparado de las políticas migratorias de tres países de la costa del Pacífico Suramericano -Colombia, Perú y Ecuador- en los últimos cinco años (2015-2019). Esta selección se dio especialmente por tres razones: en primer lugar, se trata de los principales países receptores de migrantes venezolanos, siendo Colombia el primer país de recepción, seguido de Perú y Ecuador, respectivamente; en segundo lugar, los gobiernos en los tres países recientemente han utilizado la

\footnotetext{
* Universidad de Chile, Chile. Correo electrónico: ngissi@uchile.cl

** Universidad de Cuenca, Ecuador. Correo electrónico: papodelalife1@gmail.com

*** Universidad Católica de Pereira, Colombia. Correo electrónico: pilar.ospina@ucp.edu.co

**** Universidad Católica de Pereira, Colombia. Correo electrónico: barbara.cardoso@ucp.edu.co

***** Universidad del Rosario, Colombia. Correo electrónico: spoloa92@ gmail.com
} 
estampida migratoria para hacer sendas críticas al gobierno de Nicolás Maduro ${ }^{1}$; finalmente, por la importancia de esos países en la creación de dos espacios multilaterales en la región para hacer frente a la crisis venezolana: el Grupo de Lima y el Proceso de Quito ${ }^{2}$.

Si bien existen algunos trabajos recientes acerca de esta problemática en la región (Blouin 2019; Gandini et al. 2019) y hay varios estudios enfocados en los países aquí analizados ${ }^{3}$, en este escrito se construye un análisis desde una perspectiva comparada, a partir de las particularidades de la migración venezolana en estos territorios y de la evolución de las medidas adoptadas por cada país, con el propósito de brindar elementos de análisis a los procesos inmigratorios con alcance subregional. En estas páginas, optamos por el enfoque comparativo-descriptivo, centrando la mirada en las políticas migratorias y en el marco jurídico e institucional de las migraciones.

En el plano metodológico, se realiza una revisión normativa y un análisis de las políticas y medidas institucionales ante la migración masiva de venezolanos en cada uno de los países. Este estudio se basa en la identificación de garantías constitucionales y legales de la defensa de los derechos de los migrantes, la evolución reciente de las diversas gestiones estatales, la incidencia de elementos de índole político y diplomática, y la identificación de tendencias y consecuencias respecto de las medidas revisadas. Por último, se procede a generar reflexiones acordes a la naturaleza y la incidencia de las políticas migratorias implementadas en los países objeto de estudio.

\section{Marco referencial: enfoques respecto de políticas migratorias}

La migración internacional es un fenómeno global complejo y diverso, con distintas escalas y dimensiones políticas, económicas y socioculturales. En los últimos años ha crecido su relevancia como tema prioritario de la agenda política internacional. Las dos últimas décadas se han caracterizado por un aumento de la inmigración intrarregional SurSur (Gissi et al. 2019) y la generación de nuevos corredores migratorios con distintos perfiles sociodemográficos.

El fenómeno migratorio ha sido abordado desde distintas perspectivas teóricas, en las que sobresalen las que enfatizan los derechos de los migrantes, las estrategias de gobernabilidad o gestión y las miradas directamente securitistas (Comisión Económica para América Latina y el Caribe [CEPAL], 2018).

El enfoque centrado en los derechos humanos es el resultado del debate de la comunidad internacional relacionado con la garantía y protección de los derechos de las personas migrantes como sujetos de derechos. Los tratados y normas internacionales ${ }^{4}$ reivindican la obligación que tienen los Estados de asegurar la vida, la dignidad, la no discriminación, el acceso a la justicia, entre otros, para una población particularmente vulnerable económica, social, política y culturalmente (Ubeda 2002; Castillo 2005; Campos y Xavier da Silva 2015). Estas propuestas plantean un dilema importante para los Estados (de origen y de destino), para la soberanía y para las concepciones tradicionales del ejercicio de los derechos (Gzesh 2008; Castillo 2005). La universalidad, indivisibilidad e interdependencia de los derechos humanos trascienden y superan la condición de ciudadanía y se anteponen a la naturaleza del ser humano y de la persona (Gzesh 2008; Muñoz 2009). En coherencia, los Estados en los procesos de toma de decisiones deben incluir la formulación de políticas migratorias integrales con una visión de equidad, complementariedad e interculturalidad, con políticas públicas afines a la gestión del fenómeno migratorio en la perspectiva de los derechos humanos (Ubeda 2002; Moncada y Gajardo 2015).

Además, el enfoque securitista, fundamentado en el control y la seguridad nacionales, implica una profunda desconfianza hacia los extranjeros, siendo la migración vista como un problema en los países de destino, sobre todo cuando se produce una inmigración masiva e irregular. Este abordaje pone en evidencia las tensiones sociales generadas por la inmigración y la ausencia de procesos de integración-asimilación de los migrantes en las sociedades de acogida (Portes 2012), los que redundan en retóricas y prácticas xenofóbicas. Bajo esta perspectiva, se enfatizan los costos económicos de la inmigración que el Estado debe asumir, bien como los impactos negativos en el mercado laboral provocado por el flujo migratorio, ahondando el desempleo y los bajos salarios ${ }^{5}$. En cambio, suele ignorarse el aporte económico y cultural de los migrantes a las localidades de origen, así como su contribución a la dinámica de la economía y de la reproducción social en las sociedades de destino. Sin embargo, estudios (Collier 2013) indican que 
no hay evidencia sólida de que se incrementen los niveles de desempleo ni de criminalidad en los países receptores.

Debido a estos distintos enfoques y posiciones en el sistema-mundo (Wallerstein 2007), se ha construido una agenda migratoria conocida como el enfoque instrumental o de gobernabilidad migratoria. $\mathrm{Al}$ reconocer el contexto actual caracterizado por la globalización neoliberal, ese abordaje plantea que la migración debe encauzarse y gestionarse como parte de ese sistema. De ahí el lema de esa perspectiva, auspiciada por organizaciones internacionales -como la Organización Internacional para las Migraciones (OIM) y el Alto Comisionado de las Naciones Unidas para Refugiados (ACNUR) - y entidades gubernamentales, de buscar una migración ordenada, segura y regular, la que beneficia a todos si es bien gerenciada (Mármora 2005; Ghosh 2008; OIM 2009). Este enfoque busca lograr en teoría un equilibrio entre el derecho a migrar de las personas y la soberanía nacional de los gobiernos, y se respalda en buena medida en la lógica costo-beneficio, bajo una visión pragmática, que reconoce la imposibilidad de controlar eficazmente los flujos migratorios (Domenech 2013; Geiger y Pecud 2010; Estupiñan 2013; Pereira 2019).

En esa línea, las migraciones son consideradas mayormente en términos de "ventajas", acentuando las oportunidades que ofrecen y su capacidad de transformación en el crecimiento económico, el desarrollo social y la reducción de la pobreza. Sin embargo, las migraciones también revelan las deficiencias de los mecanismos de inclusión socioeconómica de los Estados. Las personas migrantes suelen constituir un segmento de población relegado por las políticas públicas y por los sistemas de protección social en particular. Esta situación frena los esfuerzos promovidos a favor de una mayor igualdad de derechos y de fortalecimiento de la democracia, por lo que se requiere identificar alternativas en las políticas de protección social, como puerta de entrada para la garantía de derechos de las personas migrantes.

Los gobiernos aquí analizados han tratado a la migración venezolana bajo el enfoque del gerenciamiento de las migraciones antes descrito, pero con un aditivo adicional: la caracterización de dicho fenómeno como "éxodo migratorio" o "crisis migratoria". Bajo estas expresiones hay una narrativa de fracaso del "socialismo del siglo XXI" y una estrategia para captar fondos internacionales para hacer frente a la llegada masiva de venezolanos. Ese discurso es también revestido con el adjetivo de humanitario: "emergencia humanitaria", "asistencia humanitaria", y finalmente "visa humanitaria" (Ramírez 2020), pero muchas veces viene acompañado de acciones de control y seguridad, como expulsiones, imposición de visa de ingreso, militarización o cierre de fronteras.

\section{Del silencio a la acción: las paradojas de la respuesta del Estado colombiano a la migración venezolana}

El flujo creciente de personas desde Venezuela -un millón setecientos setenta y un mil ciudadanos al 31 de diciembre de 2019 (Migración Colombia 2020) - ha demandado del Estado colombiano una respuesta de emergencia con la obligación de revisar sus estrategias en materia migratoria y trazar un camino hacia una política pública integral. Colombia históricamente se ha caracterizado por ser un país expulsor de población migrante y refugiada, de manera que sus esfuerzos en este sentido se orientaban a dar respuesta a las necesidades de los colombianos en el exterior. Por eso, la actual coyuntura constituye un gran desafío institucional para el Estado colombiano en términos de atender un fenómeno sin precedentes para el país.

El 2015 representa un primer momento de inflexión en la agenda migratoria reciente. La deportación masiva de colombianos y colombo-venezolanos demandaron del gobierno central acciones puntuales de control y monitoreo en las zonas de frontera de La Guajira, Norte de Santander y Arauca, sumadas a medidas de atención humanitaria. Asimismo, en ese momento el gobierno del expresidente Juan Manuel Santos (2010-2018) mantenía una postura "blanda" con relación a las medidas adoptadas por Venezuela y las circunstancias en las que ocurrieron las deportaciones de ciudadanos colombianos. Esa estrategia diplomática se explicaba por la coyuntura interna del país, en negociaciones de paz adelantadas con las Fuerzas Armadas Revolucionarias de ColombiaEjército del Pueblo (FARC-EP), en las que Venezuela figuraba como uno de los países garantes del proceso (Louidor 2018). Esta podría ser identificada como una de las respuestas tempranas al asunto de la migración venezolana hacia Colombia, cuyo primer objetivo fue la atención de emergencia a una población de retorno fundamentada en los lineamientos de la Ley 1565 de 2012, la que reglamenta el retorno de los colombianos residentes en el extranjero. 


\section{VENEZOLANOS EN COLOMBIA}

\begin{tabular}{|cccccccccc|}
\hline 1964 & 1973 & 1986 & 1993 & 2005 & 2015 & 2017 & 2018 & 2019 & 2020 \\
\hline 16.224 & 15.000 & 23.276 & 43.285 & 37.350 & 48.714 & 550.000 & 870.093 & $1^{\prime} 408.055$ & $1 ' 771.237$
\end{tabular}
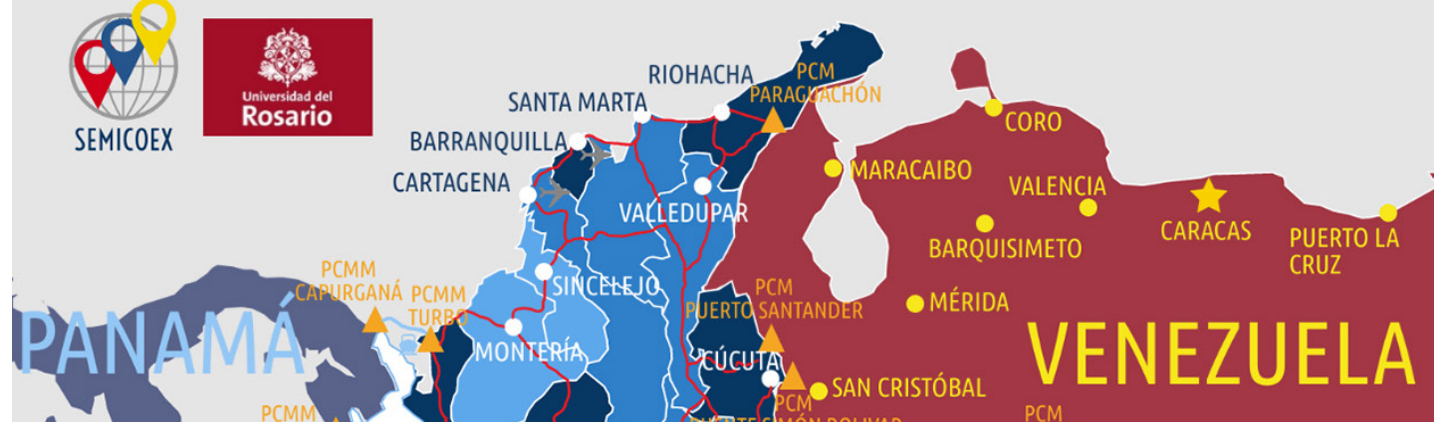

c
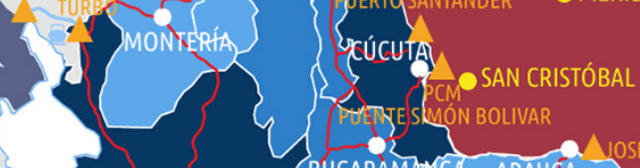

VENEZUELA

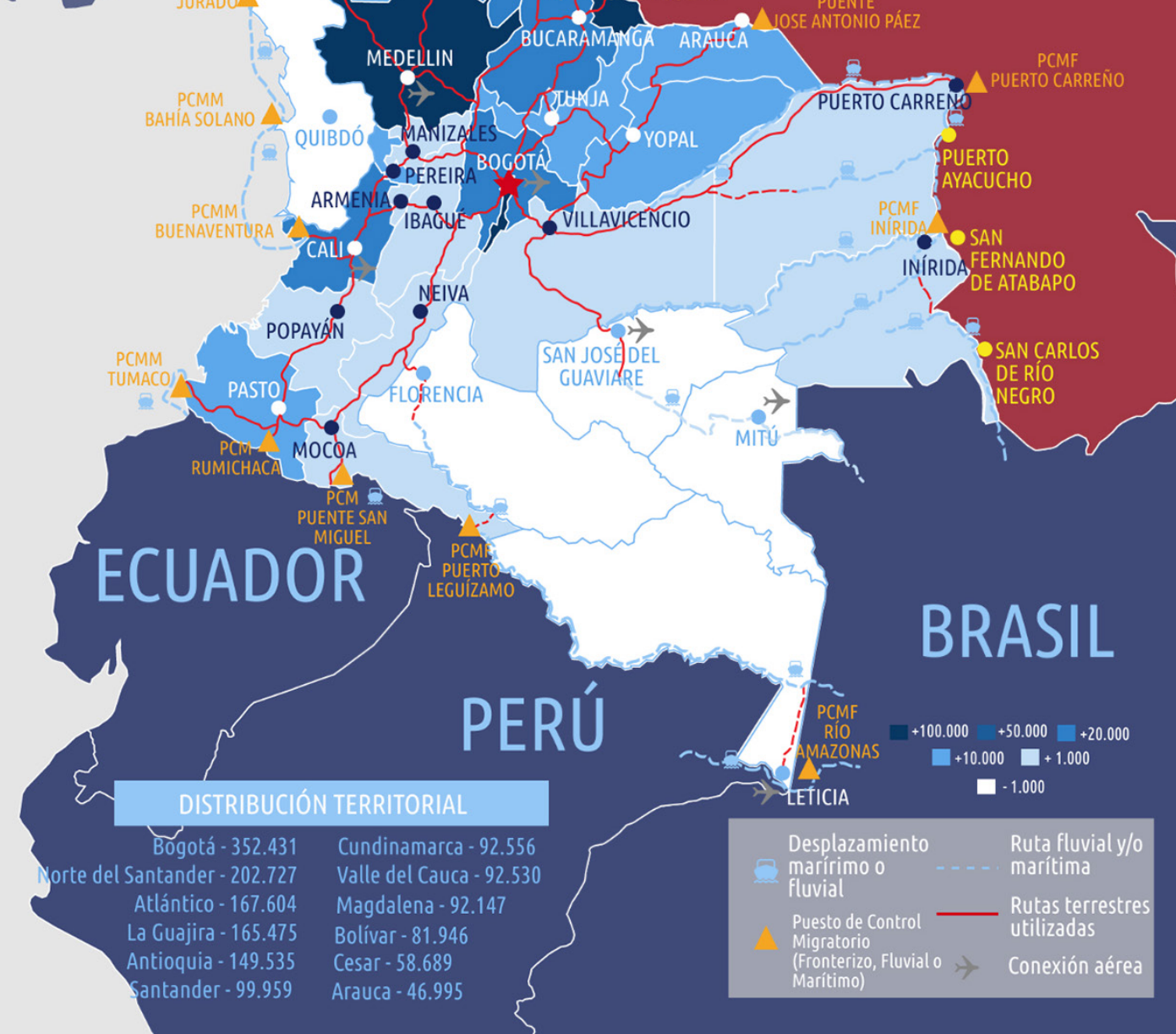

Mapa 1. Evolución, dispersión territorial y rutas de la migración venezolana en Colombia. 
En febrero de 2017, ya con los acuerdos de paz con las FARC-EP firmados y en marcha, el gobierno colombiano canaliza más atención a la agenda migratoria y adopta un conjunto de medidas direccionadas específicamente a la población migrante venezolana. Al respecto, se crean la Tarjeta de Movilidad Fronteriza (TMF), focalizada en los venezolanos que viven en las zonas de frontera, y el Permiso Especial de Permanencia (PEP), para regularizar la situación de migrantes venezolanos con intención de residencia en el país, garantizándoles el acceso a servicios básicos. Ese segundo momento de la respuesta colombiana se alinea al mejoramiento de la gestión de esta diáspora en procura de focalizar los servicios de atención básicos. Aunado a esto, en marzo de 2018 la administración de Santos también lanza los primeros pasos hacia una respuesta más estructural y de largo plazo al crear el Registro Administrativo de Migrantes Venezolanos en Colombia (RAMVC), como un "insumo para el diseño de una política integral de atención humanitaria" (Decreto 542 del Departamento Administrativo de la Presidencia de la República) (Louidor 2018).

Paradójicamente, el gobierno colombiano también adopta un conjunto de acciones para reforzar el control de las fronteras, aumentando los operativos de seguridad. En febrero de 2018 pone en operación el Grupo Especial Migratorio (GEM) en la frontera colombo-venezolana, conformado por la Policía Nacional, el Instituto Colombiano de Bienestar Familiar (ICBF), el Departamento de Impuestos y Aduanas Nacionales (DIAN) y Migración Colombia, para controlar espacios públicos, el contrabando, el uso de la TMF y del PEP, y llevar a cabo medidas de protección de menores, lo que parece camuflarse en una estrategia de militarización y contención de la migración venezolana en la frontera. Estas acciones coinciden también con un endurecimiento en la narrativa diplomática del gobierno central hacia la crisis política de Venezuela.

A cuatro días de finalizar el mandato, el expresidente Santos toma una importante decisión en torno al registro y focalización de los migrantes venezolanos para garantizar su acceso a la oferta institucional estatal. Mediante el Decreto 1288 de agosto de 2018, se reglamentan las condiciones legales de permanencia, regularización y de acceso a servicios, como salud, educación, trabajo y atención de niños, niñas y adolescentes en los distintos niveles territoriales (nacional, departamental y municipal).
Este procedimiento agilizó la designación de los PEP en el país como parte de las respuestas a los migrantes en situación irregular, un elemento que no se encontraba muy definido ni reglamentado en la legislación vigente (Ciurlo 2015).

Con la llegada de Iván Duque al poder en 2018, la diplomacia blanda en relación con el gobierno de Nicolás Maduro no se ha mantenido. Sin embargo, su discurso frente a la migración está alineado a la retórica de la solidaridad con el pueblo venezolano, reconociendo a los migrantes como víctimas del sistema político y económico venezolano. Hay una continuidad en las decisiones tomadas por su predecesor y se reitera el apoyo que Colombia brindará a los venezolanos que decidan ingresar o transitar por el país, asumiendo los retos que representan para el Estado colombiano.

En coherencia con lo anterior, la política de mediano plazo se consolida en el CONPES 3950 de 2018. Este documento tiene como objetivo general la atención a la población migrante y el fortalecimiento de la institucionalidad, a partir de la sumatoria de acciones y de responsabilidades de las entidades estatales responsables por la oferta de servicios básicos. Así, la articulación institucional se promueve como parte de la integralidad de las acciones de atención a la población migrante y desde un enfoque territorial.

La definición de las políticas de mediano plazo compiladas en el CONPES 3950 se acompaña, como se ha mencionado anteriormente, de decisiones coyunturales que responden a los desafíos inmediatos de ese fenómeno migratorio. Por eso, en noviembre de 2018, Migración Colombia establece los requisitos para el trámite de la Tarjeta de Movilidad Fronteriza para el ingreso temporal de ciudadanos venezolanos con el propósito de abastecerse de medicamentos, alimentos o incluso para que los menores de edad tengan acceso a servicios de educación primaria ${ }^{6}$. Todas estas respuestas se han dado en un marco de tensiones diplomáticas entre los dos gobiernos que a su vez se traducen en decisiones directas respecto de la gestión de las fronteras.

Aunque la mayoría de las respuestas institucionales por parte de Colombia ha sido coyuntural e inmediatista, las respuestas de corto y mediano plazo han sido insuficientes. Las presiones de la situación en las estructuras institucionales del Estado colombiano han sido de tal magnitud que han desembocado en la urgencia de establecer los lineamientos de una ley migratoria de largo plazo. 
El 23 de julio de 2019 el proyecto de ley para establecer una política integral migratoria fue radicado en la secretaría del Senado. De los aspectos a destacar se pueden mencionar la articulación con los instrumentos internacionales en materia de derechos humanos y las normas vigentes directamente relacionadas con esta cuestión. En términos procedimentales tiene por objetivo resolver lo atinente a los trámites migratorios de nacionalidad, visados, pasaportes, derechos, deberes y protección de los migrantes.

Con relación a los objetivos de largo plazo, los elementos más relevantes tienen que ver con la integración de la normativa migratoria colombiana para abarcar no solo una visión más incluyente en las atenciones a los migrantes, sino también conceptos que centran el debate en los derechos a la unificación familiar, la apatridia y los derechos de nacionalidad para los niños de padres extranjeros que nazcan en el territorio colombiano (Benavides, 2019; Morales, 2019). Estas dos cuestiones son importantes en la medida en que propone una visión abierta de la política de gestión migratoria ajustada a los cánones internacionales. Si bien la ley procura organizar el sistema de atención a los flujos migratorios desde Venezuela, es al mismo tiempo una respuesta a los procesos de emigración, inmigración y tránsito por el territorio colombiano.

\section{El giro en la política migratoria ecuatoriana a partir de la llegada de migrantes venezolanos}

El presidente de Ecuador, Lenin Moreno, en el informe de gestión de su segundo año de mandato (2019), señaló que revisaría la Ley Orgánica de Movilidad Humana, aprobada a inicios de 2017, para establecer una visa de entrada a ciudadanos venezolanos al tenor de lo que han realizado otros países de la región. Con este anuncio se terminó de cuajar el giro que dio el gobierno con relación a la política migratoria regional, al pasar de un enfoque de garantía de derechos y facilidades de residencia hacia uno más restrictivo, de control y seguridad fronteriza.

La Constitución de la República del Ecuador (2008) es emblemática al incluir, por primera vez, varios principios innovadores en materia de movilidad humana. Aparte de reconocer el derecho a migrar, estableció el principio de ciudadanía universal, la libre movilidad de todos los habitantes del planeta, el progresivo fin de la condición de extranjero (Ramírez 2016).

Una de las primeras medidas que tomó el gobierno del expresidente Rafael Correa (20072017) fue eliminar las visas de ingreso para todas las nacionalidades del mundo en "aplicación al principio de libre circularidad de personas y con el fin de fortalecer las relaciones entre Ecuador y todos los países del mundo y promover el turismo" (Ministerio de Relaciones Exteriores y Movilidad Humana [MREMHU], 2008). A nivel internacional se buscó la firma de convenios bilaterales y multilaterales, incluso con Venezuela -el denominado Estatuto Migratorio (firmado en 2010)-. Ese instrumento establecía ciertas facilidades para la residencia temporal o permanente de los ciudadanos de ambos países.

Ahora bien, es válido señalar que los venezolanos hasta el 2015 no conformaban los principales grupos de inmigrantes en Ecuador. Según cifras del MREMHU, su crecimiento pronunciado aparece en 2016, se dispara en 2017, continúa exponencialmente en 2018 y empieza a decaer en el 2019 a partir de las políticas restrictivas impuestas por el gobierno (Ministerio de Gobierno 2019). A diferencia del escenario anterior, se observa que, desde 2016, las entradas y las salidas ya no van de la mano, lo que advierte el incremento de personas que llegan de Venezuela y se quedan en Ecuador. Sin embargo, Ecuador es principalmente un país de tránsito de esta migración (Ramírez et al. 2019; Herrera y Cabezas 2019).

Estas primeras personas que deciden asentarse en Ecuador logran hacerlo mediante la "visa convenio" prevista en el Estatuto Migratorio. En efecto, entre enero de 2013 y diciembre de 2016, el Estado ecuatoriano otorgó 27.224 visas a ciudadanos venezolanos, siendo más de 13.000 gracias al Estatuto. De ahí, le siguen las visas profesionales (5.500), de amparo (3.000) y de trabajo (2.200), aproximadamente ${ }^{7}$. Hay que destacar que, al ser las visas profesionales la segunda más solicitada, se puede tener una idea del perfil migratorio de los primeros venezolanos, muchos con altos niveles de escolaridad.

Con la aprobación y puesta en marcha de la nueva Ley Orgánica de Movilidad Humana en 2017, se incorporaron nuevas categorías migratorias en el Ecuador, como la ciudadanía suramericana. Bajo este concepto, impulsado desde la Unión de Naciones Suramericanas (UNASUR), se incluyeron 


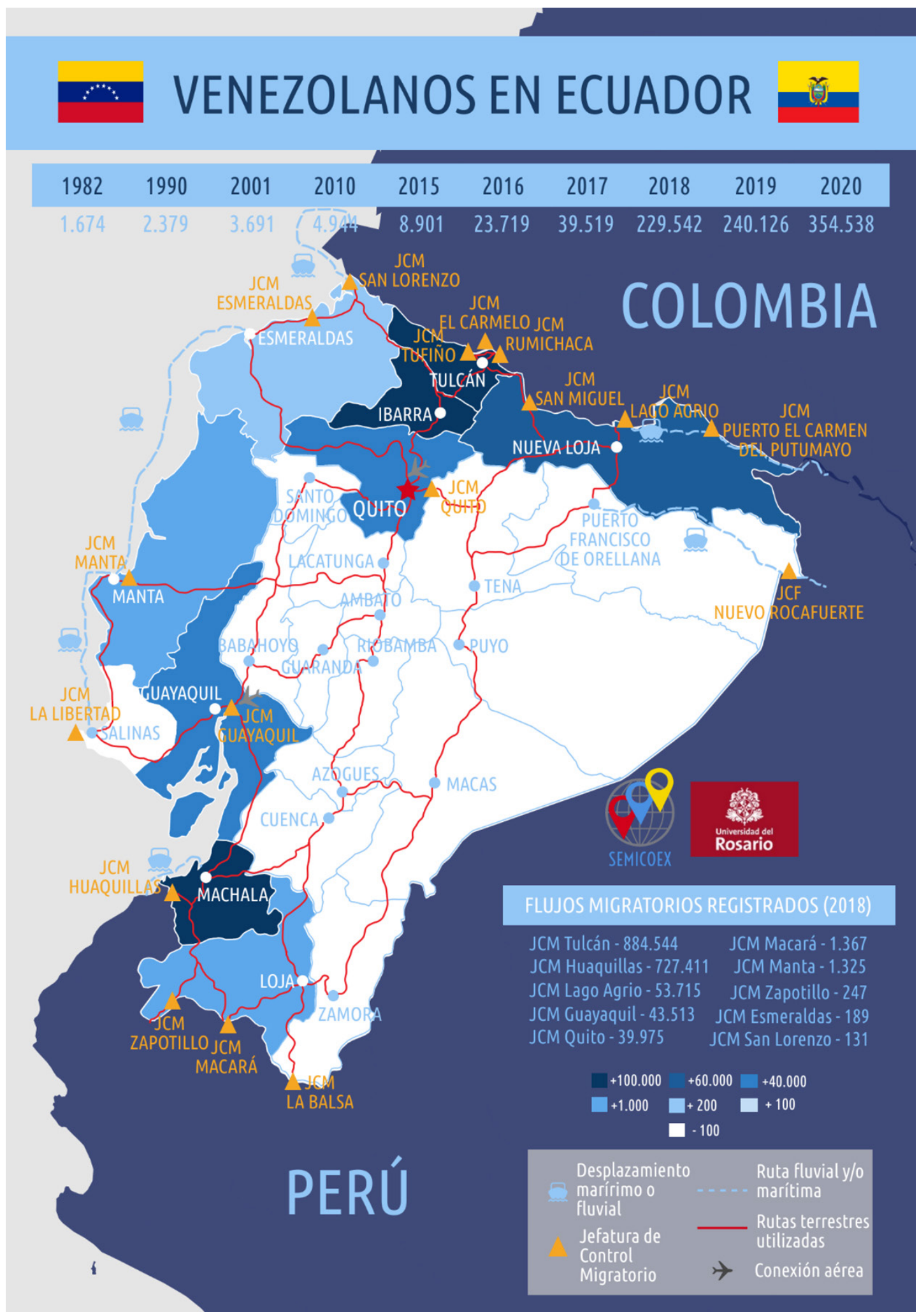

Mapa 2. Evolución, dispersión territorial y rutas de la migración venezolana en Ecuador. 
facilidades para la obtención de la residencia de las personas de la región. Ecuador constituye el único país de Suramérica que lo incluye en su marco normativo (Ramírez 2017), brindándoles facilidades a la regularización de personas migrantes, porque no se solicita ningún requisito vinculado a la actividad laboral ni a la solvencia económica del solicitante. Con la "visa UNASUR" se han regularizado aproximadamente 85.000 venezolanos hasta finales de diciembre del 2019.

Las visas convenio dejan de ser utilizadas por los venezolanos con la entrada en vigencia de la categoría de ciudadanía suramericana en 2017. A partir de esa fecha, se observa también la disminución del número de visas profesionales otorgadas, lo que sugiere, por un lado, un cambio en el perfil de los venezolanos en arribar al país y, por otro lado, la dificultad por obtener ciertos documentos apostillados, como el título profesional. Además, los datos de los venezolanos que han optado por quedarse en Ecuador en los últimos dos años muestran que muy pocos son refugiados o necesitados de protección internacional (apenas el 3\%).

El número total de visas otorgadas a migrantes venezolanos, hasta el 2019, es de 112.000, de estos, el $75 \%$ fueron visas UNASUR. Las principales dificultades para regularizar han sido el costo de las visas y los obstáculos para cumplir con todos los requisitos de documentación exigidos, tales como la apostilla de ciertos documentos y las multas en caso de haber sobrepasado el tiempo en calidad de turistas (GADPP 2020). Aproximadamente $40 \%$ de la población venezolana estaría con algún tipo de permiso de residencia temporal o permanente, sin contar los que entran legalmente como turistas y tienen un tiempo limitado en tal condición. Datos proporcionados por la Plataforma de Coordinación para refugiados y migrantes venezolanos $(\mathrm{R} 4 \mathrm{~V}$ 2019) señalan que en Ecuador residen 385.000 venezolanos hasta diciembre del 2019.

Como se mencionó al inicio, el gobierno de Moreno dio un giro en su política exterior y migratoria ante el incremento de las llegadas de venezolanos, especialmente a partir de agosto de 2018. En esa ocasión, se declaró situación de emergencia del sector de movilidad humana en las provincias de Carchi, El Oro y Pichincha, las de mayor tránsito migratorio, y se señaló la necesidad de "mantener el estado de alerta y operativos para realizar acciones inmediatas que se requieran; así como afrontar cualquier situación negativa que se pudiera generar por el flujo migratorio inusual" (MREMHU 2018a). Esto motivó a un nuevo Acuerdo Ministerial de Cancillería (Número 242), en el que "se establece como requisito previo al ingreso a territorio ecuatoriano la presentación del pasaporte con una vigencia mínima de seis meses previa a su caducidad a los ciudadanos de la República Bolivariana de Venezuela" (MREMHU 2018b).

A inicios del 2019 el gobierno continuó con su política securitista y responsabilizó a los migrantes venezolanos por el incremento de feminicidios en razón de un caso en el norte de Ecuador. El presidente Moreno señaló, el 20 de enero de 2019, en redes sociales: "He dispuesto la conformación inmediata de brigadas para controlar la situación legal de los inmigrantes venezolanos en las calles, en los lugares de trabajo y en la frontera. Analizamos la posibilidad de crear un permiso especial de ingreso al país". Un día después de este anuncio el Ministerio del Interior y la Cancillería ecuatoriana firmaron un nuevo Acuerdo Interministerial (Número 001) en cuyos considerandos se recuerda la facultad del Estado de registrar ingresos y salidas, inadmitir a personas extranjeras "que sean consideradas una amenaza o riesgo para la seguridad interna" (MREMHU y Ministerio del Interior 2019).

Si bien estas medidas fueron frenadas por inconstitucionales, es evidente el reforzamiento del enfoque de control y seguridad migratoria, amparados en el discurso de precautelar la migración riesgosa y los derechos de los migrantes (Castro 2018). Bajo esta óptica, una de las últimas medidas del gobierno fue declarar una "amnistía migratoria" y visa de ingreso para ciudadanos venezolanos (Decreto $\mathrm{N}^{\circ} 826$, de 2019), entregando una nueva visa desde finales de octubre de 2019, denominada "residencia temporal de excepción por razones humanitarias". Dicha amnistía duró hasta agosto de 2020.

La exigencia de visa de ingreso -considerada por el canciller de Ecuador como un gesto de "hermandad real" y "extremadamente solidaria"en la práctica se traduce en los mismos requisitos de la visa UNASUR, con la diferencia del costo (50 dólares) y la temporalidad de ingreso, debido a que solo aplica para aquellos que entraron en el país hasta el 25 de julio del 2019, mientras que la visa UNASUR es un mecanismo permanente para todos los ciudadanos suramericanos.

A esto se suma el envío a la Asamblea Nacional, a finales de julio del 2019, de un Proyecto de Ley Orgánica reformatoria a la Ley Orgánica de 
Movilidad Humana, el que confirma el giro reciente que se ha dado a la política migratoria en Ecuador, estableciendo criterios más rigurosos en torno a la seguridad, la soberanía y el control. En el referido proyecto se elimina cualquier mención a la ciudadanía suramericana y se deja abierta la posibilidad de exigirse visas de entrada: "El Estado ecuatoriano podrá establecer mecanismos temporales de autorización de ingreso para ciudadanos suramericanos ante casos de necesidad debidamente fundamentados (artículo 34, Proyecto de Ley Orgánica reformatoria), (Presidencia de la República del Ecuador 2019). De igual manera, el proyecto de ley apunta a optimizar los procesos de deportación de ciudadanos extranjeros que infrinjan la legislación.

Ese conjunto de medidas pone en evidencia que Ecuador no solo se alejó del eje bolivariano sino que también se alineó con el Grupo de Lima. Por iniciativa y bajo el liderazgo de Ecuador, se lanzó en 2018 el llamado Proceso de Quito, una instancia regional que reúne a 13 países para tratar el tema de la migración venezolana, pero que ha resultado más enfocado en la búsqueda de cooperación técnica y financiera para la "asistencia humanitaria".

\section{La institucionalidad en jaque: reformas, medidas e implicaciones de la recepción de venezolanos en Perú}

La historia de Perú desde finales del siglo $\mathrm{XX}$ ha mostrado una condición migratoria como país expulsor de personas. Los diversos procesos derivados de las crisis políticas durante las décadas de los ochenta y noventa, sumado a episodios de inestabilidad económica, violencia e inseguridad, condicionaron el crecimiento de nuevos procesos migratorios (Castro et al. 2013).

Las condiciones normativas para la migración del país devenían, inicialmente, de los lineamientos trazados por la Ley de Extranjería (Decreto Legislativo No. 703) de 1991, el que, sin reglamento de implementación, resultó "obsoleta para las características que presenta, actualmente, el fenómeno migratorio a nivel nacional, regional y global" (Koechlin 2016: 6).

Esta normativa, al ser "interpretada por el funcionario de turno siguiendo las directrices del gobierno" (Koechlin 2016: 9), terminaba por abrir espacio a la violación de derechos y libertades de los extranjeros en Perú. Además, se "imponía una serie de sanciones fallidamente reguladas, las cuales posicionaban a un gran número de personas inmigrantes en una situación de desventaja frente a los demás ciudadanos y de vulnerabilidad en cuanto al ejercicio y protección de sus derechos" (Polo et al. 2019:167).

Ante ese escenario, se promulgó la Ley de Extranjería (Decreto Legislativo No. 1.236) de 2015 , en la que se establece formalmente a la Superintendencia Nacional de Migraciones como un eje central de la gestión migratoria (Araujo y Dulanto 2016). Asimismo, se actualizan las condiciones y requerimientos que permiten una mayor facilidad en el tránsito y migración regular en Perú, y se desarrolla la construcción del Registro de Información de Migraciones como mecanismo de seguimiento estadístico del fenómeno. Empero, a pesar de que dicha normativa también implicaría la creación de un entramado de sanciones y elementos de control en los migrantes, "se eliminaron 130 requisitos innecesarios que se solicitaban para realizar diversos trámites migratorios" (Koechlin 2016: 15).

Posteriormente, a pesar del avance consolidado con el Decreto Legislativo No. 1.236 de 2015, se promulga la nueva Ley de Migraciones (Decreto Legislativo No. 1.350) de 2017, la que profundizaría los procesos establecidos por la ley antecesora. Con esta ley:

La garantía de protección de los derechos fundamentales, la consolidación de un Registro de Información de Migraciones (RIM), la consolidación del establecimiento de dos tipos de categorías migratorias de orden temporal y de residencia, entre otros factores, han significado que la política migratoria peruana ha desarrollado nuevas nociones que van más allá del aprovechamiento económico del flujo de remesas procedente de la diáspora peruana, además de condicionar los entramados legislativos necesarios para responder a nuevas realidades (Polo, Serrano y Jiménez 2019: 169).

Además, con el incremento de arribos de migrantes procedentes de Venezuela, el gobierno impulsa políticas de regularización, estas obedecen según Acosta, Blouin y Freier (2019: 11) a una estrategia reactiva mediante la implementación de instrumentos ad hoc, lo que supone la necesidad de realizar una ampliación de las acciones institucionales 


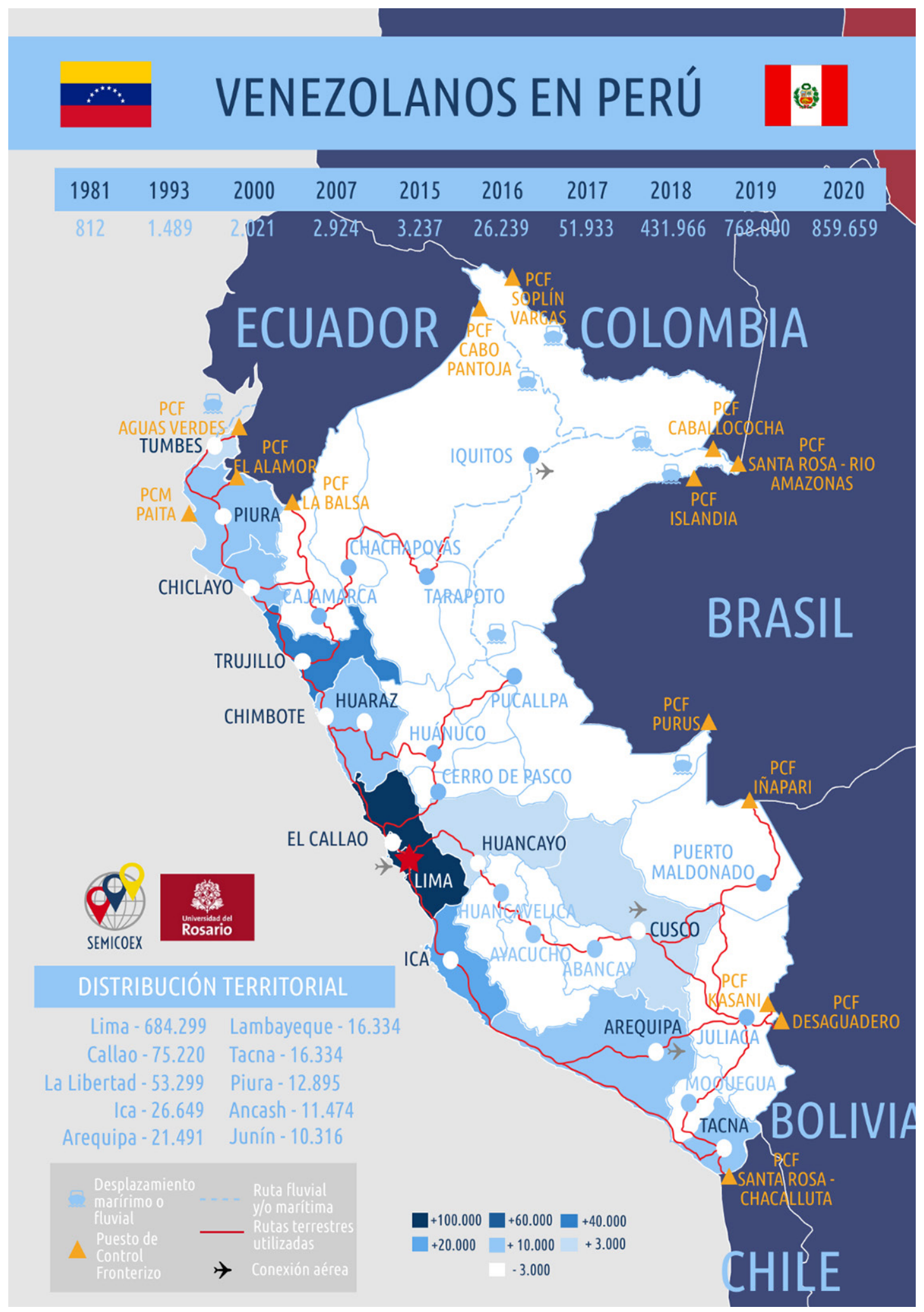

Mapa 3. Evolución, dispersión territorial y rutas de la migración venezolana en Perú. 
para atender a las grandes poblaciones de migrantes venezolanos.

El crecimiento de las poblaciones migrantes en Perú fue, en comparación con otros casos de la región, de proporciones modestas (CEPAL 2017). A pesar de que migrantes venezolanos no han figurado como principales grupos de extranjeros en el país, su presencia en Perú ha tenido un crecimiento exponencial durante la última década, pasando de registrarse 2.924 personas en el Censo de 2007, a registrarse 51.933 en el Censo de 2017 (INEI 2017). Para finales de 2019 se estimó la presencia de 859.659 venezolanos en el país (R4V 2019), lo que demanda directamente respuestas del Estado peruano frente a ese fenómeno.

El crecimiento observado en el periodo 1997 a 2017 se relacionó directamente con particularidades de la situación socioeconómica de Venezuela, sobre todo con las políticas de control de cambio instauradas por Hugo Chávez (1999-2013). El acceso cambiario limitado condicionó no solo la disponibilidad financiera de los turistas y migrantes venezolanos, sino también el rango de movimiento y la duración de los viajes en el exterior. Dichos condicionamientos, así como la limitada posibilidad de cupo de dólares para viajes y compras, derivaron en la búsqueda de espacios que permitieran aprovechar la accesibilidad de dólares en países cercanos para repatriarlos y venderlos en el mercado negro. Perú pasó a figurar como uno de los destinos para el "turismo cambiario" recurrente para los venezolanos, lo que representaría la posible consolidación de una primera concurrencia masiva de esta población durante la última década.

Más allá de la reciente modernización de las políticas de extranjería y migración, el panorama de la migración se acentuó como resultado, en parte, de la posición internacional de Perú, específicamente, por ser un país líder en la coordinación internacional de oposición al gobierno de Nicolás Maduro, como sede del Grupo de Lima. No obstante, en el plano migratorio, las capacidades estatales se han visto limitadas para responder de forma generalizada ante el flujo masivo de migrantes venezolanos. Su participación compartida con otros países en el Proceso de Quito, buscando concatenar políticamente los esfuerzos de los países miembros hacia la "atención humanitaria" a la "crisis migratoria", pone en evidencia esas dificultades.

Es necesario mencionar que se han implementado diversos mecanismos de regularización migratoria para generar una condición diversificada de la migración venezolana. La creación del Permiso Temporal de Permanencia (PTP), el uso de mecanismos de tránsito y migración de orden regional, como la Tarjeta Andina de Migraciones (TAM) y la Visa Mercosur (esa última suspendida para venezolanos en 2017), demuestran la vigencia de un entramado institucional y de cooperación internacional para responder a esta emergencia, así como de las diversas estrategias de regularización o tránsito para ingresar a Perú.

Sin embargo, el surgimiento de estos nuevos mecanismos de filtro y recepción de migrantes en Perú (sobre todo la PTP), ante las condiciones de precariedad institucional para la emisión y renovación de pasaportes por parte del Estado venezolano, ha generado limitaciones a la movilidad que inciden directamente en los migrantes venezolanos. Estos condicionamientos se han visto reflejados directamente en la frontera norte del país (principal punto de ingreso), en los pasos fronterizos de Macará, Lalamor, Jimburá, La Balsa y Huaquillas con Ecuador, y en menor medida en los demás puntos fronterizos. En junio de 2019 el gobierno del presidente Martín Vizcarra establece restricciones para la entrada al país a quienes no dispongan de pasaporte, así como la exigencia del visado humanitario como requisito sine qua non de entrada tal como quedó estipulado en la Resolución de la Superintendencia No. 0001772019. Vale señalar que Vizcarrá eliminó el PTP que implementó su antecesor. Además, se determina la necesidad de mantener un orden de registro y regularización, como en los demás casos de la región, para enfrentar el subregistro demográfico. No obstante, también los argumentos acerca de la necesidad de filtros migratorios que obedezcan a un proceso de regularización apelan al establecimiento de garantías y condiciones de seguridad para la población peruana, lo que supone un interés securitizador del Estado peruano en el control de sus migraciones.

En conclusión, a pesar de la modernización de las directrices, enfoques y mecanismos de política migratoria en Perú, la particularidad de la migración venezolana ha sido un fenómeno paralelo que, paulatinamente, se ha ido transformando en un agente activo de la agenda de reforma migratoria en Perú. En línea a las diversas modernizaciones vigentes en la normativa, el Estado peruano ha fortalecido su capacidad institucional frente a los asuntos migratorios. Por otra parte, la naturaleza de la migración venezolana en Perú se ha desarrollado por condiciones similares a la tendencia generalizada de 
la emigración de Venezuela. No obstante, también esta migración ha tenido perfiles particulares debido a la existencia de lógicas de conexión y establecimiento de redes sociales migratorias, el liderazgo político regional de Perú y las lógicas de tránsito migratorio intracontinentales de venezolanos en América Latina.

\section{Conclusiones}

La migración venezolana se ha transformado en un hecho prioritario en la agenda internacional de la subregión Pacífico Sur. Colombia, Ecuador y Perú han implementado políticas y mecanismos de regularización específicos, tales como permisos transitorios y visas especiales, aunque no han sido suficientes frente a los desafíos asociados a ese fenómeno migratorio. Si bien existe una emergente coordinación política de índole regional, hay particularidades en las estrategias adoptadas por cada país, asociadas a su trayectoria histórica en la gestión migratoria y el escenario político más reciente.

El análisis de los tres países examinados da cuenta de la implementación de un enfoque de gestión de las migraciones que ha mutado (cada vez que hay incremento de flujos migratorios) hacia enfoques restrictivos de seguridad y control, llegando hasta la militarización y cierre de fronteras.

Las políticas y legislación migratorias que se cocinan en los países analizados deberían tener el reto de responder a varios desafíos: 1) el cumplimiento de las responsabilidades de los Estados frente a la garantía de los derechos de los migrantes en sus territorios y de sus nacionales en el extranjero; 2) la adecuada respuesta a las necesidades de una población migrante, a quienes se les debe garantizar un trato digno y sobre la base del respeto a los derechos humanos, facilitándoles acceso a los servicios básicos; y 3) la adopción de estrategias frente a las reacciones de los nacionales a la inmigración, combatiendo comportamientos violentos, xenófobos y discriminatorios para tener una mejor inclusión.

Al mismo tiempo, estas políticas migratorias deberán ser una respuesta financiera y logística a las sobredemandas en la provisión de servicios que trasciendan la ayuda humanitaria. En ese sentido, la formulación de las políticas sociales con enfoque territorial se ha proyectado con el propósito de fortalecer estratégicamente las instituciones y los entes territoriales responsables de ejecutar los planes de atención básicos en coherencia con los planes de desarrollo nacional y local. Sin embargo, su eficacia de esas medidas dependerá de la articulación institucional y de la corresponsabilidad desde los distintos niveles territoriales.

Un elemento clave en la construcción de una política migratoria integral es comprender que existe un reto importante en los mecanismos de respuesta de la migración en el corto plazo, pero en la medida en que se dan procesos de integración efectiva de esta población en el mediano y largo plazo, respetando la diversidad cultural que la migración acarrea. Salir de miradas securitistas y gerenciales en torno a la migración implica superar las perspectivas hegemónicas y el reto de construir nuevos paradigmas, al menos para hacer frente a los movimientos intrarregionales, desde una visión humanista que acoja, proteja e incorpore a las personas que por cualquier motivo cruzan una frontera.

\section{Agradecimientos}

Agradecemos el apoyo del Programa U-Redes, de la Vicerrectoría de Investigación y Desarrollo (VID), Universidad de Chile. Presentamos aquí resultados del Proyecto "U-Nómades. Red de Investigación Socio-Antropológica en Migraciones, Relaciones Interculturales y Políticas Públicas" (2017-2020).

\section{Referencias Citadas}

Acosta, D., Blouin, C. y Freier, L.F.

2019 La emigración venezolana: respuestas latinoamericanas. Documento de Trabajo Agenda 2030. Fundación Carolina, Madrid.

Araujo, F. y Dulanto, P.

2016 "A propósito del Decreto Legislativo No. 1236 - Nueva Ley Migratoria”. Derecho y Sociedad 46: 341-346.

Banco Mundial

2018 Migración desde Venezuela a Colombia: impactos y estrategia de respuesta en el corto y mediano plazo. Washington.
Bastidas, C.

2020 Sistematización de estudios sobre la caracterización de la migración venezolana en Ecuador (Quito y Guayaquil). Organización Internacional del Trabajo, Quito.

Benavides, Angélica.

2019 "Cancillería creó proyecto de ley migratoria, que crea Sistema Nacional de Migración". Asuntos Legales, 24 de Julio. https:// www.asuntoslegales.com.co/actualidad/cancilleria-radicoproyecto-de-ley-migratoria-que-crea-sistema-nacional-demigracion-para-enfrentar-la-llegada-de-venezolanos-2888521 
Berganza, I. y Solórzano, X.

2019 "El proceso de integración social de la migración venezolana en el Perú". En Después de la llegada. Realidades de la migración venezolana, coordinado por Blouin, C., pp. 82-101. Thémis-PUCP, Lima.

Blouin, C.

2019 Después de la llegada. Realidades de la migración venezolana. Thémis-PUCP, Lima.

Blouin, C. y Freir, L.

2019 "Población venezolana en Lima: entre la regularización y la precariedad". En Crisis y migración de población venezolana, Entre la desprotección y la seguridad jurídica en Latinoamérica, coordinado por Gandini, L., Lozano, F. y Prieto, V., pp. 157-183. UNAM, Ciudad de México.

Bravo, G.

2016 "Las Migraciones Internacionales y la seguridad multidimensional en tiempos de la Globalización”. Diálogo Andino, 48:139-149.

Campos, B. P. C. y Xavier da Silva, J. G. L. G.

2015 "Igualdade, Não-Discriminação e Política para Migrações no Brasil: antecedentes, desafios e potencialidades para o acesso da pessoa migrante a direitos e serviços". Em Migrações, deslocamentos e direitos humanos, organizado por Galindo, G. R. B., pp. 50-63. IBDC Grupo de Pesquisa C\&DI, Brasília.

Castillo, M.

2005 Migraciones y Derechos Humanos. El Colegio de México, Ciudad de México.

Castro, Y.

2018 "Migración y fronteras en el fetichismo de la ley". Urvio 23: 29-43.

Castro, A., Hernández, C. Herrera, W.

2013 Migración y Estado en la Región Andina. Fundación Esperanza, Bogotá.

Collier, P.

2013 Inmigrantes, emigrantes y países. Turner, Madrid.

Comisión Económica para América Latina y el Caribe [CEPAL] 2018 Protección social y migración. Una mirada desde las vulnerabilidades a lo largo del ciclo de la migración y de la vida de las personas. CEPAL, Santiago de Chile

Comisión Económica para América Latina y el Caribe [CEPAL] 2017 Investigación de la Migración Internacional en Latinoamérica-IMILA [Bases de datos de censos para países latinoamericanos y del Caribe]. CEPAL, Santiago de Chile.

Ciurlo, A.

2015 "Nueva Política Migratoria colombiana". Revista Internacional de Cooperación y Desarrollo 2: 205-242.

Cuevas-Calderón, E.

2018 "Reconfiguración Social: entre la migración y la percepción de inseguridad en Lima, Perú". En Urvio 23: 73-90.

Domenech, E.

2013 "Las migraciones son como el agua: hacia la instauración de políticas de control con rostro humano. La gobernabilidad migratoria en la Argentina". Polis. Revista Latinoamericana 35: 1-21.

Estupiñán, M.L.

2013 Acerca de la gobernanza y la gestión migratoria. Crítica del papel de la OIM en el gobierno de la migración internacional y regional. Serie Documentos de Trabajo (33). Clacso, Buenos Aires.
Fundación Ideas para la Paz [FIP]

2018 Seguridad Ciudadana y migración venezolana. Análisis exploratorio, FIP, Bogotá.

Franco, J. A. y Suaza, N.

2019 "La diáspora venezolana y su repercusión en la estructura socioeconómica colombiana". Revista CEA 5:83-98.

Gámez, I., Osorio, N. A. y García, J. E.

2020 "Las calles del hambre en Ecuador: un estudio sobre la reciente migración venezolana”. Revista Colombiana de Sociología 43: 37-58.

Gobierno Autónomo de la Provincia de Pichincha [GADPP]

2020 Encuesta a las personas en movilidad humana en la provincia de Pichincha. Quito: GADPP.

Gandini, L., Lozano, F. y Prieto, V.

2019 Crisis y migración de población venezolana. Entre la desprotección y la seguridad jurídica en Latinoamérica. Ciudad de México: UNAM.

Ghosh, B.

2008 "Derechos humanos y migración: el eslabón perdido". Migración y Desarrollo 10:37-63.

Geiger, M. y Pecudo, A. (eds.).

2010 The Politics of International Migration Management. Migration, Minorites and Citizenship. Palgrave Macmillan, Hampshire.

Gissi, N., Ghio, G. y Silva, C.

2019 "Diáspora, integración social y arraigo de migrantes en Santiago de Chile: Imaginarios de futuro en la comunidad venezolana". Migraciones 47: 61-88.

Gzesh, S.

2008 "Una redefinición de la migración forzosa con base en los derechos humanos". Migración y Desarrollo 10: 97-126.

Herrera, G. y Cabezas, G.

2019 "Ecuador: de la recepción a la disuación. Políticas frente a la población venezolana y experiencia migratoria 2015-2108”. En Crisis y migración de población venezolana. Entre la desprotección y la seguridad jurídica en Latinoamérica, coordinado por Gandini, L., Lozano, F. y Prieto, V., pp. 125-155. UNAM: Ciudad de México.

Human Rights Watch.

2018 El éxodo venezolano. Urge una respuesta regional ante una crisis migratoria sin precedentes. Recuperado de: https:// www.hrw.org/es/report/2018/09/03/el-exodo-venezolano/ urge-una-respuesta-regional-ante-una-crisis-migratoria-sin Instituto Nacional de Estadística e Informática [INEI].

2017 Censo Nacionales 2017. XII segundo de Población. INEI: Lima.

Koechlin, J.

2016 Informe OBIMID - Ley de Extranjería en el Perú: Antes y Después, Observatorio Iberoamericano sobre Movilidad Humana, Migraciones y Desarrollo, Lima.

Louidor, W. E.

2018 "La migración forzada venezolana a Colombia (20152018): de una revisión documental a los esbozos de un análisis coyuntural y estructural". En El éxodo venezolano: entre el exilio y la emigración, editado por Koechlin, J. y Eguren, J. Colección OBIMID, Vol. 4.

Mármora, L.

2005 Las políticas migratorias internacionales, OIM-PAIDOS, Buenos Aires.

Migración Colombia

2018 Comunicado oficial. Desde hoy los ciudadanos venezolanos podrán tramitar la tarjeta de movilidad fronteriza $(2018,27$ de 
noviembre). Recuperado de : http://www.migracioncolombia. gov.co/index.php/es/prensa/comunicados/comunicados-2018/ noviembre-2018/8937-desde-hoy-los-ciudadanos-venezolanos-podran-tramitar-la-tarjeta-de-movilidad-fronteriza

Migración Colombia

2020 El flujo creciente de personas desde Venezuela - un millón setecientos setenta y un mil ciudadanos al 31 de diciembre de 2019 (2020, 2 de febrero). Recuperado de https://www.migracioncolombia.gov.co/noticias/ mas-de-un-millon-setecientos-setenta-y-un-mil-venezolanosestan-radicados-en-colombia-migracion-colombia

Ministerio de Gobierno Ecuador

2019 Flujo de ciudadanos de todas las nacionalidades. Recuperad de https://www.ministeriodegobierno.gob.ec/ migracion/

Ministerio de Relaciones Exteriores y Movilidad Humana

[MREMHU]

2008 Boletín de Prensa No 398, Quito.

MREMHU

2018a Acuerdo Ministerial № 152 . Viceministerio de Movilidad Humana, 9 de agosto del 2018, Quito.

\section{MREMHU}

2018b Acuerdo Ministerial No 242. Viceministerio de Movilidad Humana, 16 de agosto del 2018, Quito.

MREMHU y Ministerio del Interior

2019 Acuerdo Interministerial $N^{\circ} 001,21$ de enero del 2019, Quito.

Moncada, C y Gajardo, Y.

2015 “Interculturalidad, migrantes y Educación”. Diálogo Andino 47: 3-6.

Morales, Cindy.

2019 "Radican proyecto de ley para establecer política migratoria colombiana". 24 de julio. El Tiempo. https://www.eltiempo.com/mundo/venezuela/ proyecto-de-ley-para-establecer-politica-migratoria-colombiana-fue-radicado-en-el-congreso-392540

Muñoz, J,

2009 "Derechos Humanos, migraciones y ciudadanía universal". Diálogos Migrantes 2: 8-20.

Oficina del Alto Comisionado de las Naciones Unidas para los

Derechos Humanos [OACNUDH]

2018 Violaciones de los Derechos Humanos en la República Bolivariana de Venezuela: una espiral descendente que no parece tener fin. Recuperado de: https://www.ohchr.org/ Documents/Countries/VE/VenezuelaReport2018_SP.pdf

Organización Internacional para las Migraciones (OIM)

2009 Aspectos básicos para la gestión migratoria, OIM-INM, ciudad de México.

Penchaszadeh, A. y García, E.

2018 "Política migratoria y seguridad en Argentina hoy: ¿el paradigma de derechos humanos en jaque?". Urvio 23: 91-109.

Pereira, A.

2019 "El nexo entre migración, seguridad y derechos humanos en la política migratoria de Argentina (1990-2015)". Desafíos 31:273-309.

Pinto, L., Amaya, P. y Aliaga F.

2019 "La integración de los venezolanos en Colombia en los ámbitos de la salud y la educación". En Espacio abierto: cuaderno venezolano de sociología 28 (1), 199-223.

Polo, S., Serrano, E., y Jiménez, S.

2019 "Las migraciones colombianas hacia Perú: la invariabilidad de los flujos migratorios en un período de auge de la diáspora (2005-2015)". Revista Ciencia Política 24: 143-174.

Presidencia de la República del Ecuador.

2019 Proyecto de Ley Orgánica reformatoria a la Ley Orgánica de Movilidad Humana, 30 de julio del 2019. Quito

Portes, A.

2012 Sociología económica de las migraciones internacionales, Anthropos, Barcelona.

Ramírez, J.

2020 "De la ciudadanía suramericana a la visa humanitaria: el cambio de rumbo en la política migratoria y la diplomacia ecuatoriana". Estudios Fronterizos (manuscrito entregado en abril 2020).

Ramírez, J. Linares, Y. y Useche, E.

2019 "(Geo) Políticas migratorias, inserción laboral y xenofobia: migrantes venezolanos en Ecuador". En Después de la llegada. Realidades de la migración venezolana, coordinado por Blouin. C. Ed. Themis-PUCP, Lima.

Ramírez, J.

2018 "De la era de la migración al siglo de la seguridad: el surgimiento de las "políticas de contro con rostro (in) humano"”. Urvio 23: 10-28.

Ramírez, J.

2017 "Lo crudo, lo cocido y lo quemado: Etnografía de la ley de movilidad humana de Ecuador". En Migración, Estado y Políticas, compilador por Ramírez, J. . Vicepresidencia de Bolivia-CELAG, La Paz.

Ramírez, J.

2016 "Migration Policy in the New Ecuadorean Constitution. Toward the Formation of a Transnational Nation-State". Latin American Perspectives 43: 175-186.

R4V.

2019 Plataforma de Coordinación para refugiados y migrantes venezolanos. https://r4v.info/es/situations/platform/ location/7512

Rodríguez, R. y Ramos, F.

2019 "Colombia de cara a los desafíos y oportunidades que representa la migración venezolana". En La crisis venezolana: impactos y desafíos, editado por Pastrana E., Gehring, H., pp. 547-577. Fundación Konrad Adenauer, Bogotá.

Úbeda, G.

2002 "Políticas públicas, protección de los migrantes y relación con el derecho internacional de los derechos humanos". En Primer curso de capacitación para organizaciones de la sociedad civil sobre protección de Migrantes, editado por Instituto Interamericano de Derechos Humanos. Recuperado de https://www.iidh.ed.cr/IIDH/media/2035/i-cursocapacitacion-organizaciones-proteccion-migrantes-2002. pdf

Wallerstein, I.

2007 Geopolítica y geocultura. Ensayos sobre el moderno sistema mundial, Kairós, Barcelona. 


\section{Notas}

1 Con el rompimiento de relaciones diplomáticas con Venezuela y el reconocimiento de Juan Guaidó como Presidente Encargado de Venezuela.

2 Grupo de Lima conformado por doce países de América: Argentina, Brasil, Canadá, Chile, Colombia, Costa Rica, Guatemala, Honduras, México, Panamá, Paraguay y Perú desde el 8 de agosto del 2017 para encontrar salida a la crisis en Venezuela. El Proceso de Quito que reunió a trece países, pero cuya primera declaración fue firmada por once gobiernos de Latinoamérica: Argentina, Brasil, Chile, Colombia, Costa Rica, Ecuador, México, Panamá, Paraguay, Perú y Uruguay. El Plan de Acción por ocho. No lo firmaron de los anteriormente nombrados México, Brasil y Panamá.

3 Para el caso colombiano ver: Pinto, Amaya y Aliaga (2019), Rodríguez y Ramos (2019), Franco y Suaza (2019), Louidor (2018), FIP (2018). Para el caso ecuatoriano, ver: Ramírez, Linares y Useche (2019), Herrera y Cabezas (2019), Gámez, Osorio y García (2020), Bastidas (2020). Para el caso peruano, ver: Blouin y Freier (2019), Berganza y Solórzano (2019), Koechlin, J. (2016).
4 Algunos ejemplos en esta materia es la Convención sobre el Estatuto de los Refugiados (1951), la Declaración de Cartagena (1984), la Convención Internacional sobre la Protección de los Derechos Humanos de todos los Trabajadores Migratorios y sus Familiares (1990) y la Declaración de San José (1994).

5 Acerca de ese enfoque en la región, ver Ramírez (2018), Cuevas-Calderón (2018), Penchaszadeh y García (2018), Bravo (2016).

6 Es importante señalar que este documento se expide con la finalidad de facilitar los movimientos migratorios pendulares para evitar el paso por puntos no autorizados, pero es un documento que no es homologable ni a un permiso de trabajo ni a un trámite de permanencia (Migración Colombia, 2018).

7 Los datos son provenientes del Ministerio de Gobierno (MDG-VDI-SDM-DSM-2019-6951-O y del Ministerio de Relaciones Exteriores y Movilidad Humana (MREMHSSMC-2020-0004-O), (MREMH-VMH-2020-0319-O). 